\title{
Non-invasive measurement of intracranial pressure in the newborn and the infant: the Rotterdam teletransducer
}

\author{
J-L Wayenberg, Ch Raftopoulos, D Vermeylen, A Pardou
}

\begin{abstract}
Knowledge of intracranial pressure may be important in many clinical situations in neonates and young infants. The best way to obtain this information would be a non-traumatic procedure. In order to test the reliability of a new fontanometer, the Rotterdam teletransducer, 25 simultaneous measurements of cerebrospinal fluid (CSF) pressure and anterior fontanelle pressure (AFP) were performed. Mean (SD) difference between CSF pressure and AFP was $-0.2(1.8) \mathrm{mm}$ $\mathrm{Hg}(95 \%$ confidence interval from -0.48 to $-0.88 \mathrm{~mm} \mathrm{Hg})$. The AFP was also measured in 60 healthy children (15 premature, 30 term newborn babies, and 15 infants). The different aspects of AFP were analysed and normal values computed. These results suggest that the Rotterdam teletransducer gives reliable continuous information about intracranial pressure and can be used in clinical practice. Interpretation of AFP plots must take the influence of postconceptional age and the physiological occurrence of pressure waves into account. (Arch Dis Child 1993; 69: 493-497)
\end{abstract}

By reducing cerebral perfusion, increased intracranial pressure may have deleterious effects on the brain. Intracranial pressure monitoring may be beneficial in many conditions such as head injury, hydrocephalus and postasphyxial, infectious, metabolic, and toxic encephalopathies. ${ }^{1}$ In infants with an open fontanelle, it is possible to measure intracranial pressure non-invasively. Several devices for anterior fontanelle pressure (AFP) measurement have appeared over the last decade, but in most techniques the applied pressure of the device on the fontanelle tends to interfere with the measured pressure. ${ }^{2}$ Moreover, the time constant of most devices is too long to permit analysis of cerebral pulse and pressure waves ${ }^{34}$ which may provide helpful information. The Rotterdam teletransducer seemed to overcome these problems. ${ }^{5}$ The purpose of this study was first to test the reliability and the repeatability of this method and second to determine normal values at various ages.

\section{Patients and methods}

PATIENTS

In the first part of the study, 25 comparative measurements between AFP and cerebrospinal fluid (CSF) pressures were performed in 18 patients in whom invasive access to CSF was required for either diagnostic or therapeutic reasons because of hydrocephalus $(n=10)$, postasphyxial encephalopathy $(n=2)$, subdural effusions $(n=2)$, encephalitis $(n=1)$, head trauma $(n=1)$, subarachnoid haemorrhage $(n=1)$, and 'idiopathic' bulging fontanelle $(n=1)$.

In the second part of the study, AFP was measured in 60 healthy infants split into four groups. Group 1: 15 premature babies (born at 28-36 weeks, aged 0-25 postnatal days and 29.5-36 postconceptional weeks); group 2: eight ex-premature babies (born at 28-36 weeks, aged 8-70 postnatal days and $37-40$ postconceptional weeks); group 3: 22 term newborn babies (aged 0-14 days); and group 4: 15 infants aged 1.5-12 months (mean $4 \cdot 6$ months). We noted the weight and head circumference and calculated the body surface area $^{6}$ of each child. During the AFP measurements, most newborns and all infants were sleeping; none was shaved. Informed consent was obtained from all patients' parents.

AFP MEASUREMENTS

The Rotterdam teletransducer was developed at the department of experimental neurosurgery. (DA de Jong et al) of Erasmus University, Rotterdam. The principles and technical details of the Rotterdam teletransducer have been extensively described by de Jong and coworkers. ${ }^{5}$ The transducer is fitted to a Perspex piston that can be moved up and down in a special tapped skull adaptor by manual rotation. The adaptor is placed on the part of the head surrounding the fontanelle and securely maintained by means of a fixation frame (fig 1). The transducer itself consists of a

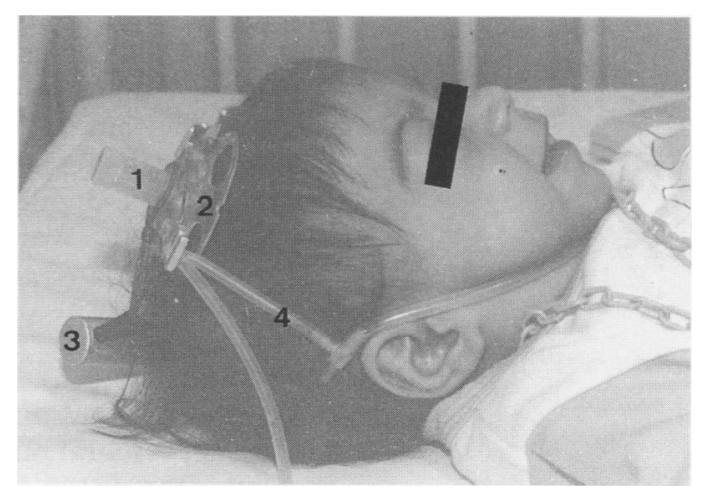

Figure 1 The adaptor with the Rotterdam teletransducer (1: piston, 2: skull adaptor, 3: oscillator-detector) fixed to the head of a child by means of a fixation frame (4). 


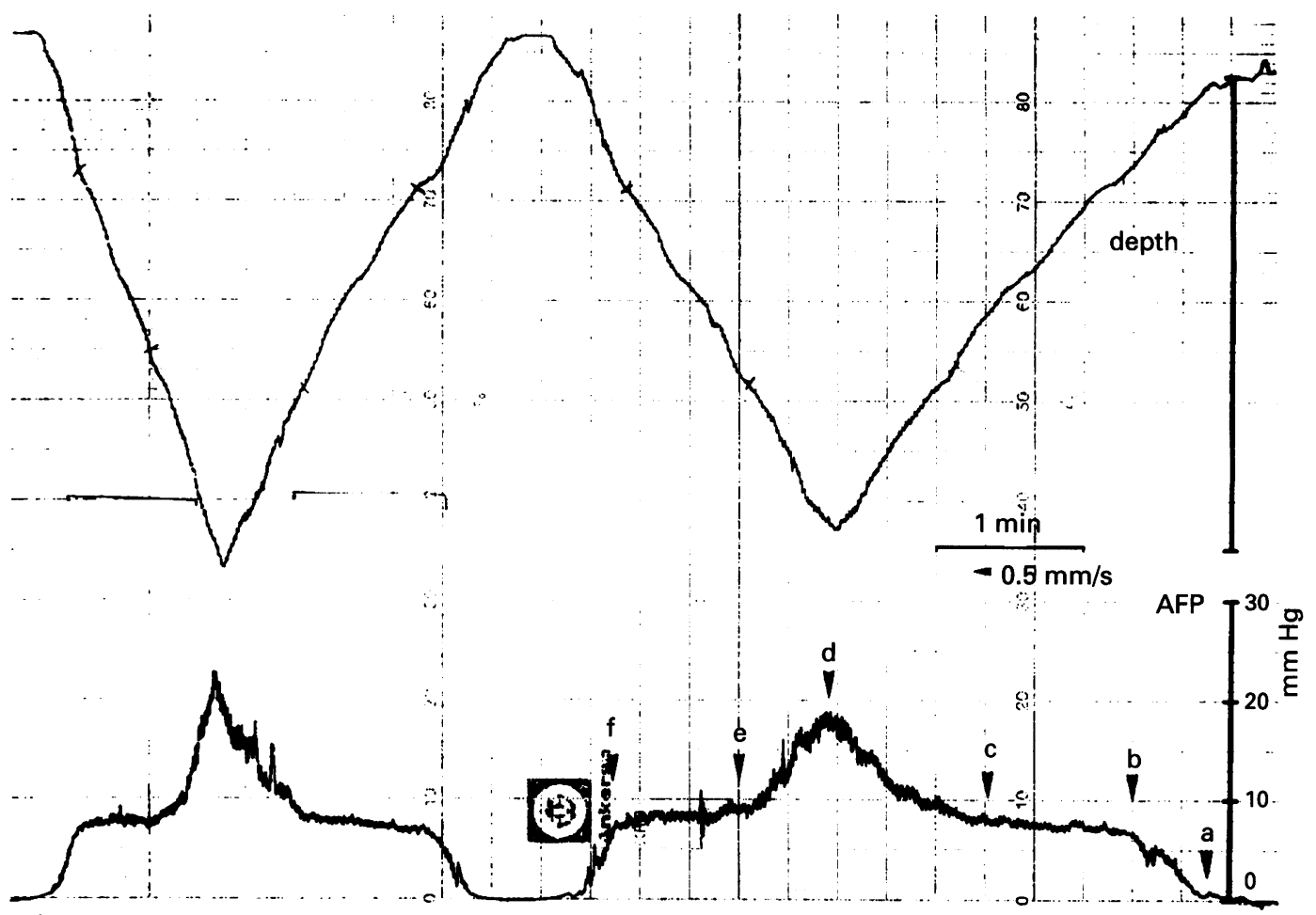

Figure 2 The pressure-depth curves. The upper trace indicates the depth of the transducer, the lower trace the measured pressure. The zero point for both traces represented initial dural contact attested by the appearance of the cerebral pulse. Slowly and gradually increasing the depth of the transducer resulted in an initial increase in the measured pressure (from point a to point $b$ ). Thereafter the measured pressure reached a plateau (from $b$ to $c$ ). Continued insertion produced a further pressure increase (from $c$ to $d$ ). Upon reaching point $d$, the resistance to manual screwing down increased. The transducer was withdrawn at an equal rate and points $e$ and $f$, corresponding to points $c$ and $b$, were obtained again. It was demonstrated that from points $b$ to $c$ (and from $e$ to f) the transducer traversed the subarachnoid space while the transducer contacted the brain tissue from points $c$ to $d$ (and from $d$ to $e$ ). ${ }^{8}$ Thus the pressure measured at the plateau corresponded to subarachnoid pressure.

sensing membrane of titanium and a passive coil capacitor circuit encapsulated in ceramic. This circuit is induced by an external oscillator. Changes in the pressure exerted on the transducer induce linear changes in the resonance frequency of the circuit. The resonance frequency is detected by an external detector by means of radio waves. The intensity of the radio waves detected decreases when the transducer moves down, so this intensity is a relative measurement of the transducer's position. In this way, both pressure and depth positioning of the transducer may be simultaneously recorded. The detector is connected to a portable monitor by means of a flexible wire which does not interfere with the child's movements. The monitor contains a measuring unit and a calibration circuit which automatically compensate for changes in atmospheric pressure and temperature. Calibration of the measuring unit is checked before each recording.

Appropriate depth positioning of the transducer in the fontanelle is done by turning the piston screw manually. This technique is based on the pressure-depth-time response described by Schettini and Walsh. ${ }^{8}$ The pressure-depth curve (fig 2) represents the response of the transducer (measured in pressure) to progressive pushing of the piston on the fontanelle (measured in depth). The zero setpoint procedure is simple: by pushing the piston on the fontanelle, cerebral pulse appears on the chart; thereafter the piston is slowly moved up again until no pulsations of the CSF are seen on the chart. This point is the zero setpoint for pressure and depth. Thereafter, a characteristic pressure-depth curve is obtained by slowly and gradually lowering the transducer on the fontanelle. The pressure recorded at the plateau of the pressure-depth curve represents intracranial pressure. ${ }^{8}$ At least three pressuredepth curves were plotted before each AFP recording. Thereafter the transducer is lowered until the depth corresponding to the plateau and the AFP recording may be continued as long as necessary. AFP was measured in dorsal horizontal position and care was taken to avoid neck flexion.

\section{INVASIVE INTRACRANIAL PRESSURE}

MEASUREMENTS

CSF pressure measurements were intraventricular when carried out just before ventriculoperitoneal derivation $(n=13)$ or were performed by lumbar $(n=10)$ or subdural $(n=1)$ puncture or via a Rickham reservoir $(n=1)$. In all cases a clear flow of CSF was obtained. CSF pressure was measured with a water column or a Claude's manometer by a person blind to the Rotterdam teletransducer reading.

\section{REPEATABILITY ASSESSMENT}

Repeatability was assessed by reperforming the pressure-depth curves during 17 continuous AFP recordings. Mean AFP recorded just before the check was compared with mean AFP obtained just after. 


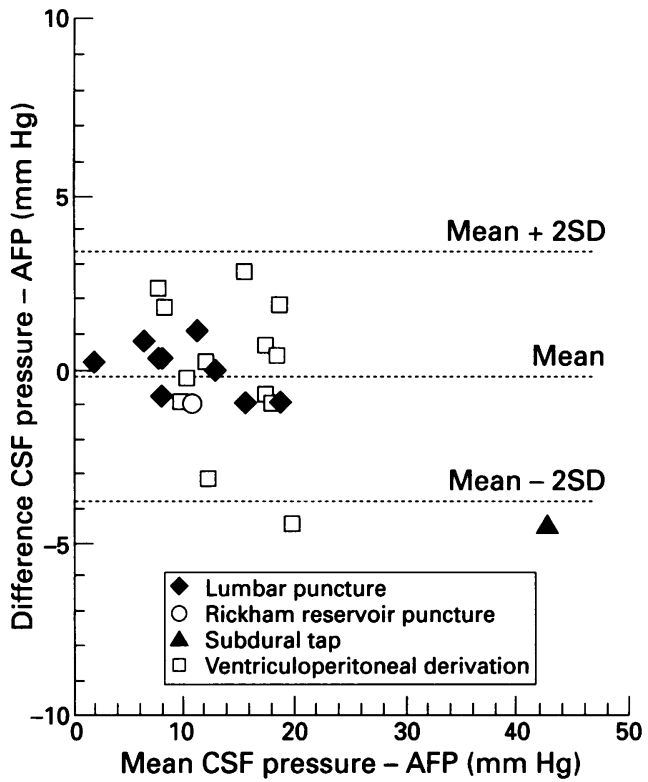

Figure 3 Differences between CSF pressure and AFP plotted against mean pressure for 25 paired measurements.

ANALYSIS OF NORMAL AFP TRACES

AFP traces were obtained with an Ankersmitt A41 recorder at $0.5 \mathrm{~mm}$ per second paper speed. We analysed only the parts of the recordings performed when the infant was quiet or sleeping. This was determined from the aspect of the AFP trace (stable baseline and absence of sudden rises in AFP suggesting movement or cry) and the stability of the depth positioning trace. AFP was calculated by the formula $\mathrm{AFP}=2 / 3$ diastolic pressure $+1 / 3$ systolic pressure. Mean AFP and mean pulse pressure amplitude were calculated as the average of points taken every five minutes, excluding movement artefacts and pressure waves. In recordings longer than one hour, we also determined the maximum AFP and the maximum pulse pressure amplitude occurring during the recording. Pressure waves are AFP increases that are not due to movement or cries, as described and adapted for children by
Paraicz. ${ }^{9}$ We noted their morphology and calculated the maximum amplitude of the pressure waves from the difference between basal diastolic pressure and peak diastolic pressure of the higher pressure wave observed during the recording.

\section{STATISTICAL ANALYSIS}

To test the reliability of the method, the differences between CSF pressure and AFP were plotted against the averages of the paired measurements. ${ }^{10}$ The same method was used to test the repeatability of AFP measurements. Differences in the AFP parameters between and within the groups were checked by Kruskal-Wallis analysis of variance and the Mann-Whitney test, respectively. Correlations between the infant's variables and AFP variables were tested by linear regression.

\section{Results}

COMPARISON BETWEEN AFP AND CSF PRESSURE MEASUREMENTS

The correlation between AFP and CSF pressure is shown on fig 3. Mean (SD) difference between CSF pressure and AFP was $-0.2(1 \cdot 8) \mathrm{mm} \mathrm{Hg}$ (extremes from -4.5 to $2 \cdot 8$ $\mathrm{mm} \mathrm{Hg}$ ). The $95 \%$ confidence interval for the bias was from -0.48 to $0.88 \mathrm{~mm} \mathrm{Hg}$.

\section{REPEATABILITY}

Mean (SD) difference of successive measurements was $0.4(0.6) \mathrm{mm} \mathrm{Hg}$ (extremes from -1.0 to $1.7 \mathrm{~mm} \mathrm{Hg}$ ). The $95 \%$ confidence interval for the bias was from 0.14 to $0.66 \mathrm{~mm}$ $\mathrm{Hg}$. The coefficient of repeatability was $1 \cdot 2$ $\mathrm{mm} \mathrm{Hg}$.

AFP RECORDINGS IN NORMAL INFANTS

One hundred and twenty six hours of AFP recording were performed on 60 normal patients (mean $126 \mathrm{~min} /$ patient, extremes 15
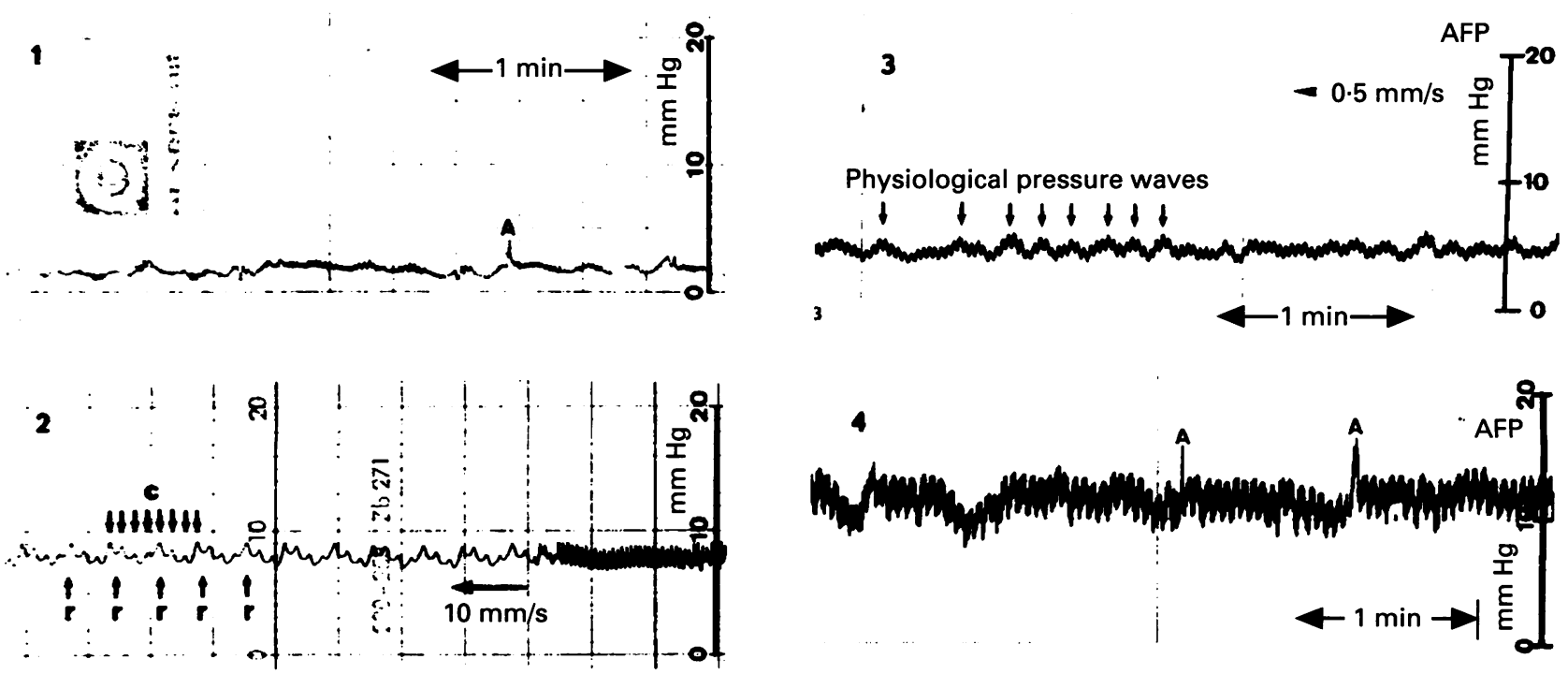

Figure 4 AFP plots from normal patients. (1) Premature neonate. (2) Term newborn. Fast paper speed (10 mm/s) shows cerebral pulse pressure components, cardiac (c) and respiratory ( $r$ ). (3) Term newborn. Note the physiological little pressure waves (arrows). (4) Infant aged 10 months. ( $A$ =artefacts.) 


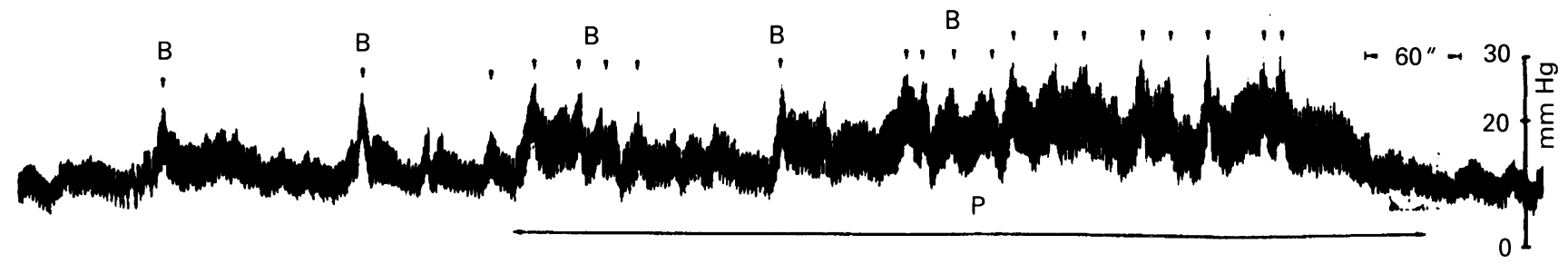

Figure 5 Sleep AFP recording in a healthy 5 month old infant showing cyclical (B-like) pressure waves (B) and a sustained (plateau) wave (P).

to $692 \mathrm{~min}) ; 74$ hours were suitable for further study (mean $74 \mathrm{~min} /$ patient, extremes 15 to 528 minutes). The AFP recording lasted more than one hour in 32 observations and more than six hours in nine.

The Rotterdam teletransducer gave high quality charts; both pulse pressure and pressure waves were clearly visible (fig 4). Cyclical AFP variations of an amplitude of $0.5-2 \mathrm{~mm} \mathrm{Hg}$ at a frequency of 2-4 waves/minute were observed in most newborns' recordings. In infants, continuous sleep recordings showed periods of cyclical pressure waves of an amplitude ranging from 1 to $15 \mathrm{~mm} \mathrm{Hg}$ and more sustained increases in AFP (fig 5). Sustained AFP waves had amplitudes ranging from 3 to $25 \mathrm{~mm} \mathrm{Hg}$ and durations ranging from 1 to 22 minutes.

Table 1 presents the mean (SD) values of the AFP variables for each group of normal children. Intergroup differences were highly significant for all AFP parameters $(\mathrm{p}<0.0001$, except for maximum amplitude of the pressure waves: $p<0.0002)$. Mean values of all AFP variables were lower in preterm than in term newborns and higher in infants than in newborns. Mean AFP and maximum amplitude of the pressure waves were well correlated with postconceptional and postnatal age (respectively $r=0.57$ and 0.52 for mean AFP; $r=0.67$ and 0.59 for maximum amplitude of the pressure waves). Also mean and maximum pulse pressure amplitude showed progressive increases and excellent correlations with regard to both postconceptional and postnatal age (respectively $r=0.89$ and 0.87 for mean pulse

Table 1 AFP variables in normal children; values are mean (SD) in $\mathrm{mm} \mathrm{Hg}$

\begin{tabular}{|c|c|c|c|c|}
\hline & $\begin{array}{l}\text { Group 1 } \\
\text { (premature) } \\
(n=15)\end{array}$ & $\begin{array}{l}\text { Group } 2 \\
\text { (ex-premature) } \\
(n=8)\end{array}$ & $\begin{array}{l}\text { Group } 3 \\
(\text { term newborns) } \\
(n=22)\end{array}$ & $\begin{array}{l}\text { Group } 4 \\
\text { (infants) } \\
(n=15)\end{array}$ \\
\hline Mean AFP & $3.1(1.5)$ & $5 \cdot 3(1 \cdot 6)$ & $5.5(1.7)$ & $7 \cdot 3(3 \cdot 0)$ \\
\hline Maximum AFP & $4 \cdot 7(1.4)$ & $7 \cdot 9(1.6)$ & $8.9(2.6)$ & $17 \cdot 0(9 \cdot 6)$ \\
\hline Mean PPA & $0.6(0.3)$ & $0.8(0 \cdot 2)$ & $0.9(0.2)$ & $2 \cdot 7(1 \cdot 3)$ \\
\hline Maximum PPA & $0.7(0.3)$ & $1.2(0.5)$ & $1.3(0.4)$ & $4 \cdot 7(2 \cdot 2)$ \\
\hline Maximum APW & $0.8(0.5)$ & $1.0(0.5)$ & $1.9(0.7)$ & $3.4(1.8)$ \\
\hline
\end{tabular}

$\mathrm{PPA}=$ pulse pressure amplitude; $\mathrm{APW}=$ amplitude of the pressure waves.

Table 2 AFP variables in normal infants before and after 4 months of age; values are mean (SD) in $\mathrm{mm} \mathrm{Hg}$

\begin{tabular}{|c|c|c|c|}
\hline & $\begin{array}{l}\text { Infants aged } \\
\leqslant 4 \text { months } \\
(n=8)\end{array}$ & $\begin{array}{l}\text { Infants aged } \\
>4 \text { months } \\
(n=7)\end{array}$ & $\begin{array}{l}\text { Significance } \\
\text { level }^{\star}\end{array}$ \\
\hline Mean AFP & $5 \cdot 7(2 \cdot 2)$ & $9 \cdot 0(2 \cdot 8)$ & $0 \cdot 007$ \\
\hline Maximum AFP & $11.0(3.9)$ & $22 \cdot 6(10 \cdot 3)$ & 0.041 \\
\hline Mean PPA & $1.9(0.8)$ & $3.5(1.2)$ & 0.003 \\
\hline Maximum PPA & $3 \cdot 2(1 \cdot 2)$ & $6 \cdot 0(2 \cdot 2)$ & 0.007 \\
\hline $\begin{array}{l}\text { Maximum APW } \\
\text { (cyclical waves) }\end{array}$ & $2 \cdot 4(1 \cdot 1)$ & $4 \cdot 1(2 \cdot 0)$ & 0.065 \\
\hline $\begin{array}{l}\text { Maximum APW } \\
\text { (sustained waves) }\end{array}$ & $3.2(1.9)$ & $10 \cdot 6(7 \cdot 2)$ & 0.038 \\
\hline
\end{tabular}

*Mann-Whitney test.

$\mathrm{PPA}=$ pulse pressure amplitude; $\mathrm{APW}=$ amplitude of the pressure waves. pressure amplitude; $r=0.92$ and 0.89 for maximum pulse pressure amplitude). However, when correlations were tested in neonates only, all AFP variables were correlated with postconceptional age ( $r$ ranging from 0.52 to 0.59 ) but not with postnatal age. Neither AFP variable was correlated with sex, weight, head circumference, and body surface area. In the neonatal groups, differences in AFP parameters between small and adequate for gestational age newborns did not reach a significant level. In infants, the values of AFP variables further increased after 4 months of age (table 2).

\section{Discussion}

In our experience differences between CSF pressure and AFP in a wide range of pressure levels and in various clinical situations were admissible for clinical purposes. The mean AFP estimated with the Rotterdam teletransducer in healthy children was lower than reported by workers who used other devices ${ }^{11-13}$ but is in agreement with direct CSF pressure measurements made in a similar population. ${ }^{14}$ This probably corresponds to the absence of applied pressure interference. Indeed the Rotterdam teletransducer's special skull adaptor overcomes this problem as the surplus applied force runs off via the skull. ${ }^{5}$ Using air insuflation and collodion to glue the device to the fontanelle, some recent fontanometers seemed also to resolve the problems of the application force and zero offset; accurate AFP monitoring was reported with such devices. ${ }^{1516}$ However, we suggest that the determination of the true level of the intracranial pressure is more accurate by performing pressure-depth curves. The sole limitations of the Rotterdam teletransducer method are a minimum fontanelle size of $11 \times 11 \mathrm{~mm}$ and the time necessary to install the device on the patient's head and perform the pressure-depth readings (15-60 minutes in healthy infants). However, the procedure for AFP measurement may be quickly performed in neurologically compromised patients. Moreover, once the Rotterdam teletransducer is in place, insertion depth may be controlled as necessary and recording may be continued as long as required. In pathological situations, we have performed some very long (up to 36 hours) recordings. Thus we confirmed and completed previous observations with the same device. ${ }^{717} \mathrm{We}$ observed similar AFP values in premature babies and infants as OverwegPlandsoen et $a l,{ }^{17}$ but lower values in term newborns. This discrepancy might be explained by differences in methodology. Indeed when Overweg-Plandsoen et al 
calculated AFP as the mean of the plateau pressures of three pressure-depth curves, we performed often continuous recordings.

Because of its very high frequency response the Rotterdam teletransducer permits precise evaluation of the pulse pressure amplitude and pressure waves. Pulse pressure amplitude and amplitude of the pressure waves may be of interest as indicators of cerebral compliance. ${ }^{18}$ Cyclical AFP variations were observed in most neonates and infants. Their morphology and frequency suggest $B$ waves as described in adults ${ }^{19}$ and adapted for infants, ${ }^{9}$ but of lower amplitude. In our experience, these cyclical pressure waves seemed to coincide with variations in heart rhythm, as reported with adult $\mathrm{B}$ waves. ${ }^{20}$ Also, sustained increases in AFP, suggesting plateau waves as seen in children with hydrocephalus or postasphyxial encephalopathy, ${ }^{2122}$ were observed in infants. As described in adults, pressure waves probably reflect changes in cerebral blood flow. ${ }^{23}$ Our observations agree with some observations in animals and adult patients suggesting the physiological occurrence of $B$ waves. ${ }^{23-25}$ However, plateau waves are always considered pathological in adults ${ }^{19}$ and, to our knowledge, have never been reported in normal individuals. Amplitude of the pressure waves is probably the principal difference between physiological and pathological intracranial pressure waves. Thus the Rotterdam teletransducer showed some poorly known aspects of the physiology of intracranial pressure. This may be of interest for early detection of abnormal brain compliance and disturbances in brain blood circulation. For instance, more information about the physiological pressure waves may improve early diagnosis of evolutive hydrocephalus, often based on the occurrence and amplitude of pressure waves detected by intracranial pressure monitoring. ${ }^{22}$

We found that not only does AFP increase with age, as other investigators have shown, ${ }^{121726}$ but pulse pressure amplitude and amplitude of the pressure waves do, too. In neonates we showed that postconceptional age rather than postnatal age must be considered in the interpretation of AFP recordings. This may be of importance in the determination of the pathological levels of the AFP variables in term and preterm babies. The reasons for this increase and for the appearance of plateau waves with age are unknown. As suggested by Overweg-Plandsoen, ${ }^{17}$ an explanation might be found in the variables of the 'steady state condition' ${ }^{\text {27 }}$ : intracranial pressure $=(\mathrm{CSF}$ outflow $\times$ resistance of arachnoid villi) + venous pressure in the superior sagittal sinus. Each of these variables may change with age but also other factors, such as the rapid head growth rate during the first months of life, the relationship of brain growth to cranial growth, the maturation of brain functions, and both progressive suture closures and reduction of the fontanelle size, may also come into play.

To conclude, the Rotterdam teletransducer allows reliable, non-invasive, and continuous measurement of the intracranial pressure. Accurate continuous information about changes in intracranial pressure and cerebral compliance may be obtained in a wide range of clinical situations without resorting to invasive techniques. As intracranial pressure is one of the determinants of cerebral blood flow, this technique may be useful in many situations accompanied with increased intracranial pressure. However, the interpretation of AFP recordings in infants must take the influence of age on AFP variables and the physiological occurrence of pressure waves into account.

1 Mann N, Punt J. Intracranial pressure monitoring in children. Fournal of the Critically Ill 1986; 2: 143-6.

2 Horbar J, Yeager S, Philip A, Lucey J. Effect of application force non-invasive measurement of intracranial pressure. Pediatrics 1980; 66: 455-9.

3 Gaab M, Sorensen N, Brawanski A, Bushe K-A, Wodarz R. Non-invasive intracranial pressure monitoring by fontanometry. $Z$ Kinderchir 1980; 31: 339-47.

4 Von Wild K, Porksen C. Non-invasive technique for monitoring intracranial pressure via the fontanelle in premature infants and newborns with hydrocephalus. $Z$ Kinderchir 1980; 31: 348-53.

5 de Jong DA, Maas A, van de Voort E. Non-invasive intracranial pressure monitoring. A technique for reproducible fontanelle pressure measurements. $Z$ Kinderchir 1984; 39: 274-6.

6 Mabry C.C. Tableau de quelques constantes biologiques normales. In: Vaughan VC, McKay JR, eds. Traité de Pédiatrie. Paris: Doin, 1980: 1964

7 Plandsoen WC, de Jong DA, Maas A, Stroink H, van Eijndhoven J. The pressure depth curve in anterior fontanelle pressure monitoring. In: Miller J, Teasdale G, Rowan J, Galbraith S, Mendelow A, eds. Intracranial prowan J, Galbraith S, Mendelow A, eds. Intracr

8 Schettini A, Walsh E. Experimental identification of the subarachnoid and subpial compartments by intracranial pressure measurements. $f$ Neurosurg 1974; 40: 609-16.

9 Paraicz E. Introduction to the 9th meeting of the International Society of Pediatric Neurosurgery. In: Paraicz E, ed. ICP in infancy and childhood. Monographs in paediatrics. No 15. Basel: Karger, 182: 1-7.

10 Bland JM, Altman D. Statistical methods for assessing agreement between two methods of clinical measurements. Lancet 1986; i: 307-10.

11 Robinson R, Rolfe P, Sutton P. Non-invasive method for measuring intracranial pressure in normal newborn measuring intracranial pressure in normal

12 Philip A, Long J, Donn S. Intracranial pressure. Sequential measurements in full-term and preterm infants. $A m \mathcal{F}$ Dis Child 1981; 135: 521-4

13 Menke J, Miles R, McIlhany M, Bashiru M, Chua C, Schwied E. The fontanelle tonometer: a noninvasive method for measurement of intracranial pressure. f Pediatr 1982; 100: 960-3.

14 Kaiser A, Whitelaw A. Normal cerebrospinal fluid pressure in the newborn. Neuropediatrics 1986; 17: 100-2.

15 Mehta A, Wright BM, Shore C. Clinical fontanometry in the newborn. Lancet 1988; i: 754-6.

16 Rochefort $M$, Rolfe P, Wilkinson A. New fontanometer for continuous estimation of intracranial pressure in the newcontinuous estimation of intracranial p

17 Overweg-Plandsoen W, de Jong DA, Hanlo P, Braun K, Avezaat $C$. Anterior fontanelle pressure values in normal young children of various age. Intracranial pressure VIII. Berlin: Springer-Verlag, 1992 (in press).

18 Avezaat C, van Eijndhoven J. Clinical observations on the relationship between cerebrospinal fluid pulse pressure and intracranial pressure. Acta Neurochir (Wien) 1986; 79: 13-29.

19 Lundberg N. Continuous recording and control of ventricular fluid pressure in neurosurgical practice. Acta Psychiatrica et Neurologica Scandinavica 1960; 36 (suppl 149): 7-193.

20 Hashimoto $M$, Higashi S, Kogure $Y$, et al. Respiratory and cardiovascular oscillations during $B$ waves. In: Hoff JT, Betz AL, eds. Intracranial pressure VII. Berlin: SpringerVerlag, 1989: 217-9.

21 Levene M, Evans D, Forde A, Archer L. Value of intracranial pressure monitoring of asphyxiated newborn infants. Dev Med Child Neurol 1987; 29: 311-9.

22 Paraicz E. A-waves in infantile and children's hydrocephalus. F Neurosurg Sci 1978; 22: 169-71.

23 Newell DW, Aaslid R, Stoos R, Reulen HJ. The relationship of blood flow velocity fluctuations to intracranial pressure B waves. $\mathcal{F}$ Neurosurg 1992; 76: 415-21.

24 Martin G, Lundberg's $B$ waves as a feature of normal intracranial pressure. Surg Neurol 1978; 9: 347-8.

25 Mautner-Huppert D, Haberl R, Dirnagl U, Villringer A, Schmiedek P, Einhaupl K. B-waves in healthy persons. Schmiedek P, Einhaupl K.
Neurol Res 1989; 11: 194-6.

26 Raju T, Doshi U, Vidyasagar D. Cerebral perfusion pressure studies in healthy preterm and term newborn

27 Marmarou A, Shulman K, Lamorgese J. Compartmental analysis of compliance and outflow resistance of the cerebrospinal fluid outflow system. I Neurosurg 1975; 43: 523-34. 